\title{
Conductas violentas en la relación de noviazgo en adolescentes
}

\section{Violent behaviors in dating relationships in adolescents}

\author{
LÓPEZ-NIEBLA, Rosa María๋*, MARTÍNEZ-CÁRDENAS, Juana María, TERRAZAS-MEDINA, \\ Tamara Isabel y MENDOZA-MIRELES, Goreti Leticia
}

Universidad Autónoma de Coahuila, Instituto de Enseñanza Abierta, Unidad Saltillo. Campos Redondos SN, Sin Nombre de Col 9, 25000 Saltillo, Coah. Méx.

ID $1^{\text {er }}$ Autor: Rosa María, López-Niebla / ORC ID: 0000-0001-7260-3300, arXiv Author ID: 8GQUZR-P8NFDZ, CVU CONACYT ID: 953192

ID $1^{\text {er }}$ Coautor: Juana María, Martínez-Cárdenas / ORC ID: 0000-0003-1004-9652, Researcher ID: Thomson: X-23702018, CVU CONACYT ID: 949979

ID $2^{\text {do }}$ Coautor: Tamara Isabel, Terrazas-Medina / ORC ID: 0000-0002-6581-190X, arXiv Author ID: 1908.09839, CVU CONCAYT ID: 929839

ID $3^{\text {er }}$ Coautor: Goreti Leticia, Mendoza-Mireles / ORC ID: 0000-0001-5191-357X, arXiv Author ID: 1908.09877, CVU CONACYT ID: 1012806

\begin{abstract}
Resumen
Los cambios actuales dan lugar a una nueva realidad social, familiar, económica, política y escolar, está ultima es el resultado de elementos multifactoriales que han cambiado la dinámica de trabajo en las aulas, la disiciplina, el ambiente escolar y las formas de aprendizaje. Por lo que el docente debe ser capaz de utilizar estrategias didácticas capaces de desarrollar habilidades en los estudiantes para que respondan a la diversidad y heterogeniedad de la sociedad de hoy. El objetivo de este estudio fue identificar las estrategias didácticas utilizadas por los docentes de bachillerato que propician aprendizajes significativos, acordes al saber aprender, saber hacer, saber ser y saber convivir, y describir las características de un buen docente. Se realizó una investigación cuantitativa de corte transversal que se aplicó a 126 estudiante de bachillerato, el 41.7\% fueron hombres y el 57.5 fueron mujeres. La selección de la muestra fue por oportunidad. Los resultados muestran que las estrategias que más utililizan son la actitud positiva, la exposición, el compromiso, el dinamismo y el trabajo colaborativo como estrategias en su didáctica. Las características que más destacan en un buen maestro son: capacidad para enseñar, inteligente, trabajador, estricto honesto y responsable.
\end{abstract}

Estrategias didácticas, aprendizaje significativo, Educación Media Superior

\begin{abstract}
Current changes lead to a new social, family, economic, political and educational reality, the educational one is the result of multifactorial elements that have changed the dynamics of classroom work, the discipline, the school environment and ways of learning. So the teacher should be able to use teaching strategies able to develop skills in students to respond to the diversity and heterogeneity of today's society. The goal of this study was to identify the teaching strategies used by high school teachers that promote significant learning chords to learn to learn, know-how, know how to be and how to live, and describe the characteristics of a good teacher. A quantitative crosssectional research was applied to 126 high school students; $41.7 \%$ were male and $57.5 \%$ were women. The sample selection was by chance. The results show that the strategies that are the most used by the teachers are: positive attitudes, persuasion, commitment, exposition and collaborative work. The features that stand out in a good teacher are: ability to teach, intelligent, hardworking, strict, honest and responsible.
\end{abstract}

Strategies, Meaningful Learning, School Education

Citación: LÓPEZ-NIEBLA, Rosa María, MARTÍNEZ-CÁRDENAS, Juana María, TERRAZAS-MEDINA, Tamara Isabel y MENDOZA-MIRELES, Goreti Leticia. Conductas violentas en la relación de noviazgo en adolescentes. Revista de Sociología Contemporánea. 2019. 6-20: 1-6.

\footnotetext{
* Correspondencia del Autor (correo electrónico: rosy_lopez_niebla@ @otmail.com)

$\dagger$ Investigador contribuyendo como primer autor.
} 


\section{Introducción}

Cada día aumenta la violencia de todos tipos en el mundo, las estadísticas mundiales reportan que la violencia más preocupante es la que se da calladamente en el seno de los hogares, donde paradójicamente es la familia el núcleo de la sociedad, el lugar donde se aprende a socializar, donde se aprenden los valores, a amar, a expresar afectos, a respetar, y, sin embargo, la violencia también es aprendida como vía de solución de los problemas.

Así, la violencia se convierte en una herramienta que implica el uso de la fuerza para producir un daño a otra persona. Organismos internacionales, nacionales y locales, han centrado sus esfuerzos en estudiar la violencia usada como control y poder en la adolescencia, período de la vida en que se descubre el amor tierno, es decir, en el noviazgo.

Numerosos estudios se han generado sobre la violencia en el noviazgo, por ser ésta predictiva de la violencia conyugal, la cual se establece en la relación íntima y estable entre un hombre y una mujer casados o en unión libre, ejerciéndola de manera física, psicológica, económica y sexual, a través de distintos grados de frecuencia y daño, dé ahí la relevante necesidad de acercarnos a este problema social, que trae consigo impactos severos en otros ámbitos de la vida.

El Instituto de Enseñanza Abierta U.S. de la Universidad Autónoma de Coahuila, en el Departamento de Tutorías y Orientación, aborda este tema en tutorías grupales e individuales, así como también en la Escuela para Padres, complementando con la aplicación de un instrumento para evaluar la violencia en el noviazgo. Nuestro objetivo es detectar, identificar y prevenir en nuestros estudiantes la violencia en el noviazgo.

\section{Conceptualización}

La violencia en el noviazgo es "todo acto, omisión, actitud o expresión que genere, o tenga el potencial de generar daño emocional, físico o sexual a la pareja afectiva con la que se comparte una relación íntima sin convivencia ni vínculo marital”.
Adolescencia según la OMS, “ la adolescencia es el período de la vida en el cual el individuo adquiere la capacidad de reproducirse, transita de los patrones psicológicos de la niñez a la adultez y consolida su independencia económica", por su parte, (Domínguez, 2008:74) señala que "los significativos cambios biológicos, por una posición social intermedia entre el niño y el adulto, en cuanto a estatus social, ya que el adolescente continúa siendo un escolar, depende económicamente de sus padres, pero posee potencialidades psíquicas y físicas muy semejantes a las de los adultos".

Noviazgo "una relación diádica que involucra interacción social y actividades conjuntas con la implícita o explícita intención de continuar la relación hasta que una de las partes la termine o se establezca otro tipo de relación como la cohabitación o matrimonio" (Straus, 2004:792).

En tanto que el Banco Mundial (2003) conceptualiza el noviazgo como "una vinculación que se establece entre dos personas que se sienten atraídas mutuamente; representa una oportunidad para conocerse, una etapa de experimentación y de búsqueda, con actividades, gustos y pensamientos en común, y es un preámbulo para una relación duradera”.

Enamoramiento "Es un estado de ánimo, puede ser un desencadenante de la experiencia amorosa hacia otra persona. Científicamente se dice que es un proceso bioquímico que inicia en la corteza cerebral, pasa a las neuronas y de allí al sistema endocrino, dando lugar a respuestas fisiológicas intensas; parece ser que sobreviene cuando se produce en el cerebro una sustancia conocida como feniletilamina".

En la Encuesta Nacional de Violencia en las Relaciones de Noviazgo, realizada por el Instituto Mexicano de la Juventud en 2007, los resultados señalan que $15.5 \%$ de los mexicanos de entre 15 y 24 años con relaciones de pareja ha sido víctima de violencia física, $75.8 \%$ ha sufrido agresiones psicológicas y $16.5 \%$ ha vivido al menos una experiencia de ataque sexual; dicho estudio destaca que la violencia en el noviazgo tiende a pasar desapercibida por las instituciones y por los propios jóvenes, lo que ocasiona la falta de apoyo institucional y familiar a las víctimas (Poy, 2008). 
En nuestra ciudad, Saltillo, Coahuila, caracterizado por ser una población de tradiciones arraigadas y pacíficas, está presentando violencia conyugal, tal y como reporta el Periódico Zócalo en su emisión del 16 de enero de 2016, con la noticia de que en lo que va del año se han presentado cuatro feminicidios. Existen diferentes teorías que explican la violencia, una de ellas es la desarrollada por Martin Sligman, llamada Indefensión aprendida, misma que el autor, indica que cuando la persona es sometida a constante estrés por situaciones de violencia, pierde la capacidad de defenderse, debido a una adaptación a tal situación. Esto debido a un deficiente juicio crítico para percibir pros, contras, consecuencias de la situación por la que se está pasando. Es entonces cuando la víctima de agresiones y violencia de cualquier tipo, es invadida por pensamientos derrotistas, como el de "así me tocó", "es mi cruz", "para qué, si no se puede hacer nada".

Por lo anterior, la violencia produce efectos o que marcan a la persona que sufre de violencia, en la adolescencia, toda vez que esta etapa se caracteriza por la búsqueda de identidad, toda vez que en esta etapa de amor tierno, el temor a perder el afecto y compañía del novio/a, propicia que las manifestaciones de maltrato se minimicen y se perciban como normales, sin tomar conciencia de que impactan en la autoestima de la víctima, debido a que el maltrato va trascendiendo de lo privado a lo público. Hay que comentar que la violencia en las relaciones de pareja no depende del sexo, pues el hombre también sufre de violencia, solo que por prejuicios machistas no lo hace saber.

Por lo cual, la violencia en el noviazgo merece especial atención, sobre todo, cuando se inician las relaciones entre los y las jóvenes y se definen roles y límites.

Tipos de violencia en el noviazgo son:

- Física.

- Psicológica.

- Sexual.

- Patrimonial.

- Económica.
La manifestación de la violencia es cíclica, pasa por cuatro episodios:

1. Fase de Acumulación de Tensión: Se manifiestan insultos, reproches, escenas de celos y control, silencios prolongados, burlas, malestar constante y en aumento.

2. Fase de Estallido o Explosión: Se manifiestan incidentes con todo tipo de agresión física o psicológica, golpes, ruptura de objetos y amenazas.

3. Distanciamiento: Hay un tiempo de silencio o separación.

4. Fase de arrepentimiento o luna de miel: Se manifiestan expresiones de perdón, promesas de cambio, interés sexual, regalos y "tranquilidad".

El abuso reiterativo se alterna con devoción sacrificada, alimentada con la fantasía de la víctima de que es realmente valorada de entre todos/as las demás personas que compiten por el amor y atención del agresor, sobreviniendo un sentimiento de culpa por haber sido la responsable del rompimiento temporal y por pensar en dejar a su agresor, toda vez que éste le ha prometido modificar su comportamiento y fortalecer su relación.

\section{Metodología}

Como se hizo referencia en el apartado de la Introducción, inspira el tema gracias a las sesiones de tutoría tanto individual como grupal, en las cuales se aborda la violencia y se culmina con la aplicación de una encuesta.

Para efectos de este trabajo, se consultó bibliografía, centrando nuestra atención la Construcción y prevalente. Validación de un Instrumento para Evaluar Violencia en la Relación de Pareja Melissa García Meraz, Elízabeth Georgina Vargas Carcía, Rebeca Guzmán Saldaña, Aleyda Vizzuet Herrera y Claudia Ruíz Sánchez, Universidad Autónoma del Estado de Hidalgo, Unidad especializada en la Prevención y atención de la violencia familiar y sexual. De su revisión, diseñamos un instrumento para evaluar si existe o no la violencia en el noviazgo en nuestros estudiantes de preparatoria e identificar su tipo prevalente. 


\begin{tabular}{|l|r|}
\hline \multicolumn{1}{|c|}{ Factor } & No. De items \\
\hline Fisica (FIS) & 5 \\
\hline Social y sexual (SOCYS) & 6 \\
\hline $\begin{array}{l}\text { Violencia y manipulacion } \\
\text { (VYMA) }=10\end{array}$ \\
\hline $\begin{array}{l}\text { Violencia economica y emocional } \\
\text { (VEYE) }\end{array}$ \\
\hline
\end{tabular}

Tabla 1

Debido a que los estudios que se han realizado sobre la violencia en el noviazgo adolescente, señalan que: es la adolescencia, la edad más vulnerable para sufrir violencia, que se mantiene en silencio porque se cree que son conductas normales, que la ejerzan generalmente los hombres, sin embargo las mujeres también utilizan formas de control como la manipulación y el chantaje, que puede darse al poco tiempo de iniciar el noviazgo y seguramente continuará en caso de que lleguen a casarse, que se da en todos los estratos sociales, que la pareja violenta es muy probable que tenga antecedentes de violencia en su familia y su conducta no cambiará espontáneamente. Que se Inicia con la violencia psicológica para después pasar a la física y luego a la sexual.

Y siendo la escuela un agente socializador, se pretende con este estudio sobre la violencia en el noviazgo adolescente indagar si ¿Existe violencia en las relaciones de noviazgo adolescente en la ciudad de Saltillo, Coahuila? Y ¿qué tipo de violencia predomina en las relaciones de noviazgo adolescente en la ciudad de Saltillo, Coahuila?

Es una investigación exploratoria el instrumento consta de 36 ítems, distribuidos en 6 factores cuyas conductas manifiestan algún tipo de violencia en el noviazgo. Los factores considerados son violencia física, social y sexual, violencia y manipulación, violencia económica y emocional. El instrumento se aplicó en el Instituto de Enseñanza Abierta, US, en la Preparatoria Mariano Narváez G. TM, en la Preparatoria Gabino García, de la Ciudad de Saltillo, Coahuila, todas ellas del nivel medio superior, tanto a hombres como mujeres, se aplicaron 102 encuestas.

\section{Resultados}

Los resultados se presentan en los cuatro factores mencionados anteriormente.
Como podemos observar en el Gráfico 1, de los cuatro factores de violencia, sobresale la violencia económica y emocional causada por el hombre hacia la mujer.

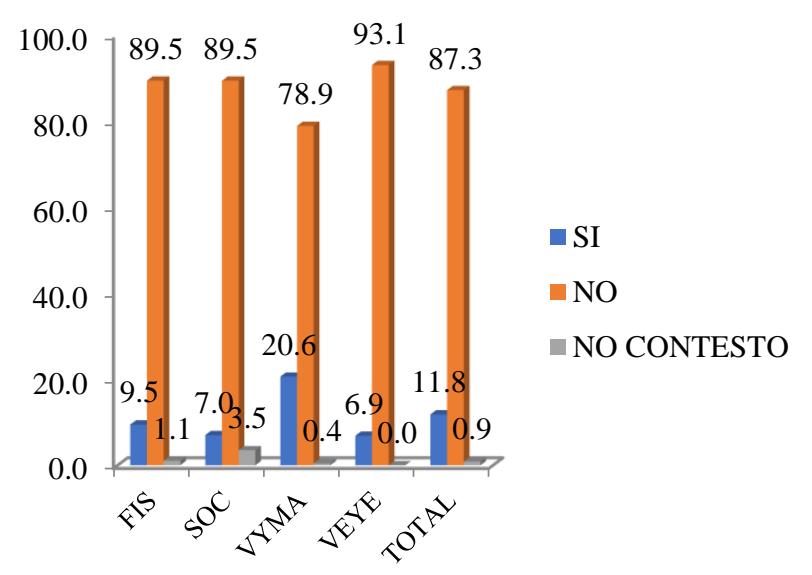

Gráfico 1 Factores de violencia

De acuerdo con el Gráfico 2, se ve un claro ejercicio de violencia económica y emocional, así como una tendencia de abuso social y sexual por parte de la mujer hacia el hombre.

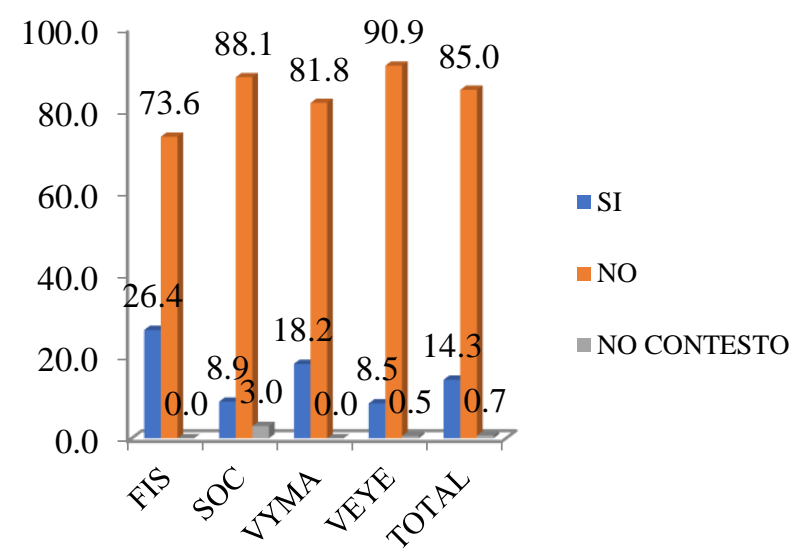

Gráfico 2 Grado de violencia de la mujer hacia el hombre

\section{Conclusiones}

Lo que pudimos concluir con esta investigación es que una gran mayoría conoce el significado de la violencia en el noviazgo, pero no todas o todos están dispuestas y dispuestos a admitir una relación de violencia, debido a que se justifican en el hecho de que solo ha pasado alguna vez. Debemos de estar conscientes del hecho de que aun cuando solo haya pasado un episodio de violencia se considera como Violencia en el Noviazgo, y se deben tomar decisiones en las cuales se valore a la relación y a las personas involucradas en ella. 
Un punto importante en este tema es que se debe entender que la Violencia en el Noviazgo no solo proviene del hombre hacia la mujer, sino también puede venir de la mujer hacia el hombre, por lo cual se debe de poner mucha atención entre la línea de la confianza y de la falta de respeto. Todas las mujeres y hombres tenemos el derecho de elegir a la persona con la que queremos estar, pero nunca debemos de perder de vista el hecho de que nuestra integridad y nuestra persona van antes de cualquier relación o persona que nos pone en riesgo.

Una relación debe de ser responsable amorosa y debe de haber comunicación entre las dos personas desde el principio de la relación se puede saber si esta será una relación violenta o amorosa. El patrón de abuso que sufren mujeres jóvenes en una relación de pareja es similar al que sufre una mujer maltratada adulta. La violencia generalmente se presenta en un ciclo en el que tanto el hombre como la mujer se encuentran atrapados.

Cuando nos encontramos ante una relación donde la violencia persiste, ese ciclo se repite, una y otra vez, donde la relación va construyendo un espiral y el periodo de duración entre las diferentes etapas es más rápido, modificándose el grado de violencia en cada fase, dando como resultado los diversos problemas sociales donde las principales afectadas en mayor porcentaje son las mujeres.

En el sexo femenino, prevalecen el económico y emocional, infringido hacia el hombre, toda vez que es un medio para denostar a su compañero, dañando sin lugar a dudas su autoestima. Situación similar en la prevalencia del factor de violencia económica y emocional, es ejercida a la mujer por el hombre.

Se desprende de lo anterior la relevante importancia que tienen las tutorías y la orientación en estudiantes de preparatoria, a fin de brindarles información para detectar manifestaciones de violencia en todos sus tipos, con el objetivo de prevención.

El rubro de autoestima, sería un factor para indagar su grado de daño y correlacionarlo con la violencia en el noviazgo.
Se proponen talleres de autoestima a los estudiantes y a sus padres, así como el "rescate" de los valores que en la actualidad se han perdido entre la sociedad creando la práctica continua de los "antivalores" los cuales han logrado sobrepasar niveles muy marcados entre la niñez y la juventud. De tal manera se propone crear una campaña de concientización sobre la equidad y el género para poder erradicar la mala influencia de las cosas negativas de nuestra sociedad en base a lo que investigaciones sostienen, que la violencia es aprendida desde el hogar, según la teoría Transgeneracional.

\section{Referencias}

Álvarez. (s/f) La indefensión aprendida. Tonalidad emocional. Recuperado en .https://mariangelesalvarez.com/igualdad/relaci on-de-control-o-igual/la-indefension-aprendida/ el 3 de Septiembre de 2019

Botella, M. (2004). (pp. 139-182). En T. Ibáñez, (coord..). Introducción a la psicología social. Barcelona: UOC

Domínguez, L. (2008) citado por ROJAS, J. (2013). Violencia en el noviazgo de adolescentes mexicanos: Una revisión. Revista de Educación y Desarrollo 27. http://www.cucs.udg.mx/revistas/edu_desarroll o/anteriores/27/027_Rojas.pdf

Hernández, R.; Fernández.; Baptista, P. (2006). Metodología de la investigación. $4^{\mathrm{a}}$. Edición. McGraw-Hill México.

Hurlock, E. (1991). Psicología de la adolescencia. Paidos.

Instituto Nacional de la Juventud (2008). Encuesta Nacional de Violencia en las relaciones de noviazgo 2007.resumen ejecutivo. Recuperado en https://www.imjuventud.gob.mx/imgs/uploads/ ENVINOV_2007_-

_Resultados_Generales_2008.pdf

Instituto Nacional de la Juventud (2008). Encuesta Nacional de Violencia en las relaciones de noviazgo 2007.resumen ejecutivo. Recuperado en https://www.imjuventud.gob.mx/imgs/uploads/ ENVINOV_2007_-

_Resultados_Generales_2008.pdf 
JUVENTUD, I. M. (5 de Noviembre de 2015). INSTITUTO MEXICANO DE LA JUVENTUD. Obtenido de http://www.imjuventud.gob.mx

Lorente, M. citado por Tapia, M. (2008), violencia en la pareja. la prevención desde el noviazgo. tesina. universidad del aconcagua facultad de psicología. Recuperado en

Martínez, F. (2015). violencia en el noviazgo. Recuperado en $\mathrm{http}: / /$ violenciaenelnoviasgolb.blogspot.com/

Martínez. (2015). violencia en el noviazgo. Recuperado en http://violenciaenelnoviasgolb.blogspot.com/

Melissa García Meraz', E. G. (5 de Noviembre de 2015). Unidad Especializada en la prevención y atención de la violencia familia, Universidad Autónoma del Estado de Hidalgo. Obtenido de http://www.academia.edu

Namakforoosh (2005, p.72). Metodología de la Investigación. Limusa. $2^{a}$. edición. México.

Poy,2008). citado por Ramírez, C. ; Núñez, D. (2010). Violencia en la relación de noviazgo en jóvenes universitarios: un estudio exploratorio enseñanza e investigación en psicología vol. 15, num. 2: 273-283 julio-diciembre, 2010. Instituto Tecnológico de Sonora Recuperado en http://bibliotecadigital.uda.edu.ar/12. El 31 de agosto de 2019

Poy,2008). citado por Ramírez, C.; Núñez, D. (2010). Violencia en la relación de noviazgo en jóvenes universitarios: un estudio exploratorio enseñanza e investigación en psicología vol. 15, num. 2: 273-283 julio-diciembre, 2010. Instituto Tecnológico de Sonora.

Ramírez, C.; Núñez, D. (2010). Violencia en la relación de noviazgo en jóvenes universitarios: un estudio exploratorio enseñanza e investigación en psicología vol. 15, num. 2: 273283 julio-diciembre, 2010. Instituto Tecnológico de Sonora Recuperado en http://www.conductitlan.org.mx/09_jovenesyad olescentes/Materiales/A_Violencia+en+la+relac i\%C3\%B3n+de+noviazgo.pdf el día 3 de Septiembre de 2019
Ramírez, C.; Núñez, D. (2010). Violencia en la relación de noviazgo en jóvenes universitarios: un estudio exploratorio enseñanza e investigación en psicología vol. 15, num. 2: 273283 julio-diciembre, 2010. Instituto Tecnológico de Sonora Recuperado en http://www.conductitlan.org.mx/09_jovenesyad olescentes/Materiales/A_Violencia+en+la+relac i\%C3\%B3n+de+noviazgo.pdf el día 3 de Septiembre de 2019

Rojas, J. (2013). Violencia en el noviazgo de adolescentes mexicanos: Una revisión. Revista de Educación y Desarrollo 27http://www.cucs.udg.mx/revistas/edu_desarr ollo/anteriores/27/027_Rojas.pdf

Straus, (2004) p.792. Citado por Rojas, J. (2013). El noviazgo y otros vínculos afectivos de la juventud mexicana en una sociedad con características posmodernas. Uaricha. Revista de Psicología de la Universidad Michoacana de San Nicolás de Hidalgo, vol. 10, núm. 23, 2013, pp. 120-139, Recuperado en https://www.aacademica.org/dr.jose.luis.rojas.s olis/13.pdf

Straus, 2004, p.792. Citado por ROJAS, J. (2013). El noviazgo y otros vínculos afectivos de la juventud mexicana en una sociedad con características posmodernas. Uaricha. Revista de Psicología de la Universidad Michoacana de San Nicolás de Hidalgo, vol. 10, núm. 23, 2013, pp. 120-139, Recuperado en https://www.aacademica.org/dr.jose.luis.rojas.s olis/13.pdf

Tapia, M. (2008). Violencia en la pareja. la prevención desde el noviazgo. Tesina. Universidad del Aconcagua Facultad de Psicología. Recuperado en http://bibliotecadigital.uda.edu.ar/12. El 31 de agosto de 2019 